\title{
Sensitivity and Dynamics of Bioreceptor-based Biosensors
}

\author{
JEROME S. SCHULTZ \\ Department of Chemical Engineering \\ University of Michigan \\ Ann Arbor, Michigan 48109
}

Concepts for biosensors that can be used for the continuous monitoring of biochemicals are just emerging from research laboratories. The functioning and design of most of these devices are best understood in terms of their components, which usually consist of three elements: a physical transducer, biochemical reaction, and usually some protective membranes.

Here, we will focus on the properties of reversible binding proteins, such as bioreceptors, which can provide the specificity characteristics of the device. We will consider only systems involving reversible binding because reversibility is a requirement for "reagentless" sensors, that is, systems in which there is no net consumption of any of the reactant species.

There are many possible configurations for the design of biosensors using reversible bioreceptors. The simplest type is shown in FIGURE 1a, which involves only the receptor and the analyte. This system would work if there is a measurable property change in the receptor when the analyte-receptor complex is formed; for example, when gentamicin binds to an antibody, the emission spectrum of the tryptophans in the protein (antibody) are quenched. ${ }^{1}$ The chemical reactions are

$$
\begin{gathered}
\mathrm{A}+\mathrm{R} \underset{k_{\mathrm{r}}}{\stackrel{k_{\mathrm{f}}}{\rightleftharpoons}} \mathrm{A}: \mathrm{R}, \\
K_{\mathrm{a}}=k_{\mathrm{f}} / k_{\mathrm{r}},
\end{gathered}
$$

where A - analyte, $\mathrm{R}$ - bioreceptor, A:R - analyte-receptor complex, and $K_{\mathbf{L}}=$ association equilibrium constant.

If the total amount of the bioreceptor is conserved ([R] $-[R]+[A: R])$, such as when the reaction chamber is isolated from the outside environment by a selective membrane, then the fraction of receptor in the complex form is given by

$$
[\mathrm{A}: \mathrm{R}] /[\mathrm{R}]_{\mathrm{t}}=[\mathrm{A}] K_{\mathrm{s}} /\left(1+[\mathrm{A}] K_{\mathrm{s}}\right) .
$$

A dimensionless binding curve is shown in FIGURE 2 as the solid line, whereas the expected calibration curves for hypothetical quenching efficiencies of $50 \%$ and $100 \%$ are shown as the dashed lines. It is clear that if the instrumentation does not have the capacity to subtract background or baseline fluorescence, then as the quenching efficiency decreases, the sensitivity of the assay also decreases.

An approximate numerical value for the concentration range where the sensor is operational is given by the analyte concentration required to half-saturate the 
bioreceptor, that is, when $[\mathrm{A}]-\left(K_{\mathrm{a}}\right)^{-1}$. Thus, the reciprocal of the numerical value of the association equilibrium constant provides an approximate estimate of the applicable concentration range of the sensor.

Some values for binding constants between various substrates and their respective binding proteins are given in TABLE 1 . These particular binding proteins were particularly chosen to illustrate the extreme level of sensitivity inherent in some

(a)

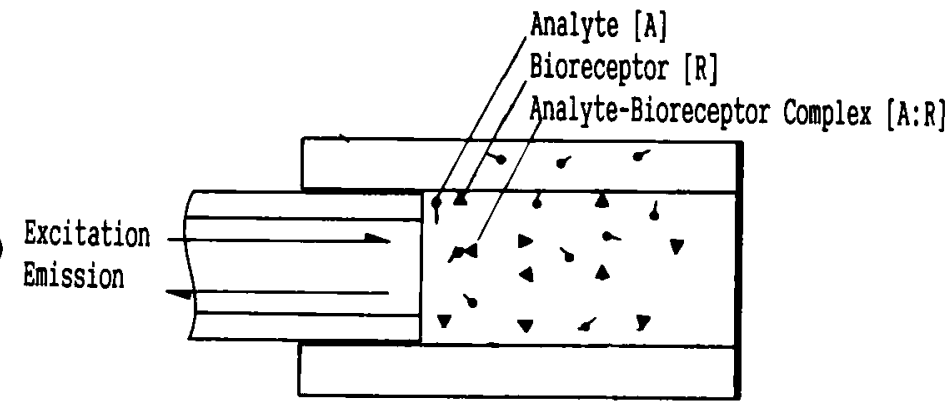

(b)

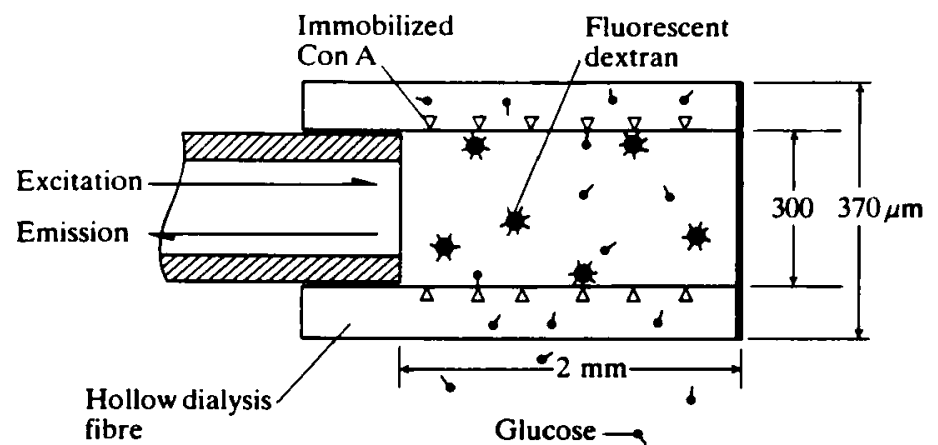

FIGURE 1. Configurations for fiber-optic biosensors based on bioreceptor binding. (a) Direct method based on the quenching of the inherent fluorescence of the bioreceptor with binding of the analyte. (b) Indirect method based on the use of a fluorescently labeled analog-analyte (FITC-dextran) that competes with the analyte (glucose) for the bioreceptor binding sites (Con-A).

biological bioreceptors, which can be seen by considering the reciprocals of the association constants in the last column, that is, $10^{-6}$ to $10^{-10} \mathrm{M}$.

In the more general situation where the direct binding of the analyte to the bioreceptor does not produce a measurable property change in the bioreceptor, then one can introduce an appropriately labeled analog-analyte into the system that will 
permit a measurement to be made. The chemical reactions are

$$
\begin{array}{cl}
\mathrm{A}+\mathrm{R} \rightleftharpoons \mathrm{A}: \mathrm{R}, & K_{\mathrm{a}}=[\mathrm{A}][\mathrm{R}] /[\mathrm{A}: \mathrm{R}], \\
\mathrm{A}^{*}+\mathrm{R} \rightleftharpoons \mathrm{A}^{*}: \mathrm{R}, & K_{\mathrm{a}}^{*}=\left[\mathrm{A}^{*}\right][\mathrm{R}] /\left[\mathrm{A}^{*}: \mathrm{R}\right] .
\end{array}
$$

The sensor shown in FigurE $\mathrm{Ib}^{2}$ is designed such that both the bioreceptor and the

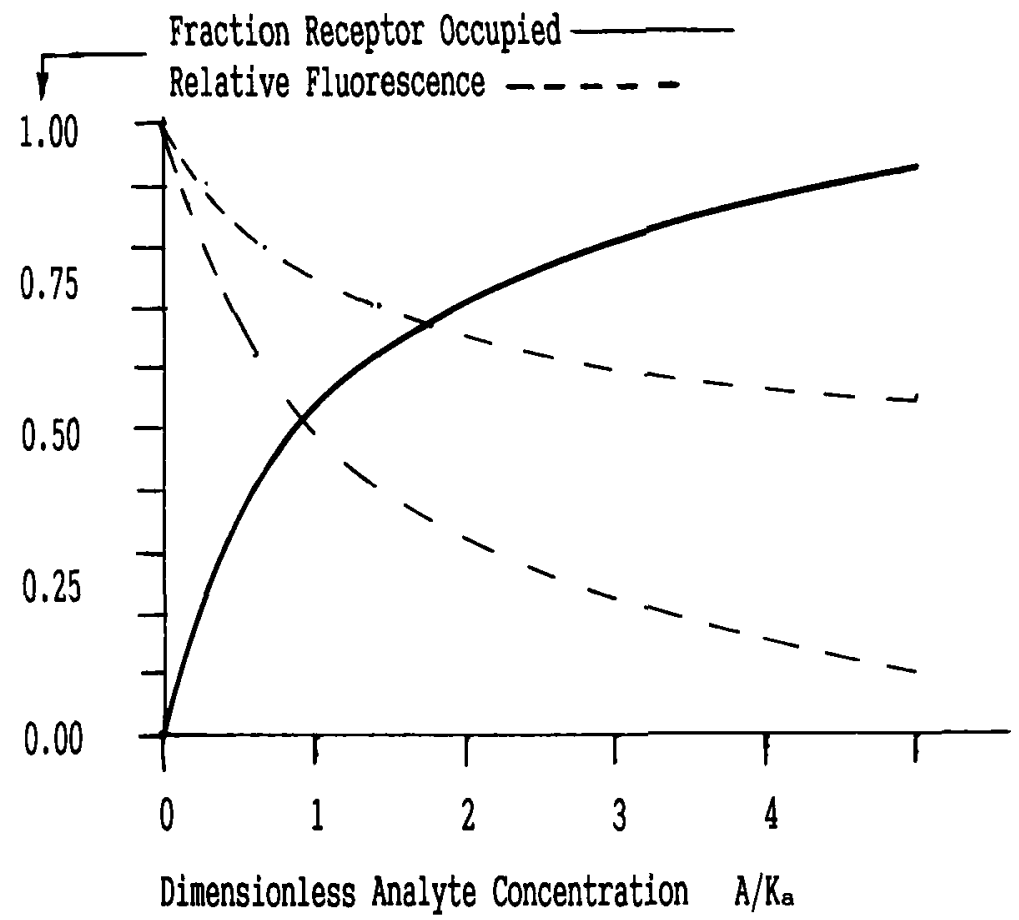

FIGURE 2. Binding curve for the monovalent complex between analyte and bioreceptor (solid curve). Expected biosensor response curves for "direct method" fluorescence quenching [dashed curves for quenching efficiencies of $100 \%$ (lower) and 50\% (upper)].

analog-analyte are conserved within the transducer, that is,

$$
\begin{gathered}
{\left[A^{*}\right]_{t}=\left[A^{*}\right]+\left[A^{*}: R\right],} \\
{[R]_{t}=[R]+[A: R]+\left[A^{*}: R\right],}
\end{gathered}
$$

where $A^{*}$ is FITC-dextran, $R$ is Con- $A$, and $A$ is glucose. The expression relating the fraction of analog-analyte existing free to total analog-analyte in the transducer chamber is given by

$$
\left(\frac{\left[\mathrm{A}^{*}\right]}{\left[\mathrm{A}^{*}\right]_{\mathrm{t}}}\right)^{2}+\frac{\left[\mathrm{A}^{*}\right]}{\left[\mathrm{A}^{*}\right]_{\mathrm{t}}}\left[\left(\frac{[\mathrm{R}]_{\mathrm{t}}}{\left[\mathrm{A}^{*}\right]_{\mathrm{t}}}-1\right)+\left(\frac{K_{\mathrm{a}}[\mathrm{A}]+1}{\left[\mathrm{~A}^{*}\right]_{\mathrm{l}} K_{\mathrm{a}}^{*}}\right)\right]-\left(\frac{K_{\mathrm{a}}[\mathrm{A}]+1}{\left[\mathrm{~A}^{*}\right] K_{\mathrm{a}}^{*}}\right)=0
$$


TABLE 1. Properties of Some Binding Protein-Ligand Systems ${ }^{9}$

\begin{tabular}{llc}
\hline \multicolumn{1}{c}{ Ligand } & \multicolumn{1}{c}{ Binding Protein } & $\begin{array}{c}\text { Association Constant } \\
\mathbf{M}^{-1}\end{array}$ \\
\hline Cyclic-AMP & $\begin{array}{c}\text { Cyclic-AMP dependent } \\
\text { protein kinase } \\
\text { Folate binding protein } \\
\text { Thyroxine binding } \\
\text { globulin }\end{array}$ & $\begin{array}{c}\left(2.5 \times 10^{7}\right. \\
\left(5 \times 10^{7}\right)-\left(9 \times 10^{9}\right)\end{array}$ \\
Thyroxine & $\begin{array}{c}\text { Intrinsic factor } \\
\text { Riboflavin binding } \\
\text { protein }\end{array}$ & $\left.6.0 \times 10^{10}\right)$ \\
Vitamin $B_{12}$ & $\begin{array}{c}7.8 \times 10^{8} \\
\text { Riboflasol binding } \\
\text { globulin }\end{array}$ & $\left(1 \times 10^{8}\right)-\left(1 \times 10^{9}\right)$ \\
Cortisol & & \\
\hline
\end{tabular}

This implicit equation is plotted in FIGURE 3 (in a dimensionless manner), and it can be seen that there are several degrees of freedom that allow one to alter or manipulate the dynamic range of sensor sensitivity to suit particular needs. This particular representation is for the situation where one is measuring the relative amount of free analog-analyte by optical methods. If instead, the system was configured to measure the amount of bound analog-analyte, then the dimensionless performance curves would be the inverse of those shown, that is, $\left(1-\left[A^{*}\right] /\left[A^{*}\right]_{2}\right)$.

Three parametric curves are shown in the figure for different ratios of the

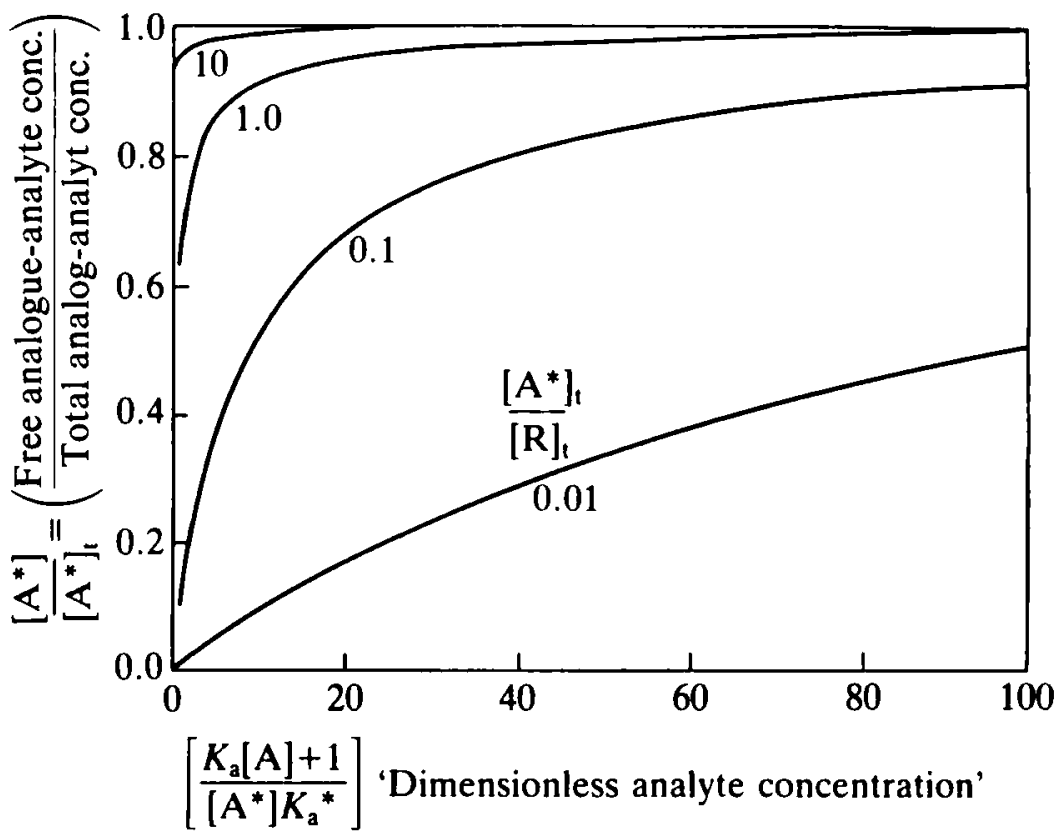

FIGURE 3. Expected behavior of biosensors based on competitive binding between an analyte, $A$, and an analog-analyte, $A^{*}$, with a bioreceptor. 
concentration of receptor sites to the concentration of analog-analyte $\left(\left[A^{*}\right]_{t} /[R]_{t}\right)$; in fact, values of this ratio between 0.01 and 0.1 would be desirable for an analytical device depending on whether the range of analyte concentrations of interest were narrow or broad.

However, even after fixing the ratio of bioreceptor to analog-analyte, the range of the device can be altered by modifying the values of the binding constants, $K_{\mathrm{a}}$ and $K_{\mathrm{a}}^{*}$. Physically, this can be achieved in a number of ways: (a) by selection of the bioreceptor, which will affect both $K_{\mathrm{a}}$ and $K_{a}^{*}$, probably to the same extent, and (b) by selection of the analog-analyte, which will affect only $K_{\mathrm{a}}^{*}$. We will discuss the latter option in some detail.

First of all, one can choose analog candidate compounds that have different intrinsic binding affinities as compared to the analyte of interest. For example, in constructing a glucose sensor, we ${ }^{3}$ used fluorescein-labeled dextran as the analoganalyte. This polymer has pendant glucose residues that bind to Con-A with an affinity on the same order as glucose binding. However, other sugars have different affinities for Con-A as shown in TABLE 2. Thus, if polymers were constructed with other sugars as pendant groups, then the value of $K_{a}^{*}$ could be changed considerably; for example, as with polymannans.

Another approach to altering $K_{a}^{*}$ with a given ligand is to alter the number of binding groups (valence) of the analog-analyte. For example, Mansouri ${ }^{4}$ has found that the apparent binding constant of dextrans to Con- $A$ is a function of the molecular weight of dextran. We use the term, "apparent" binding constant, for multivalent ligands because there is not a unique method for describing the mechanism of multivalent binding. A number of models have been proposed for this phenomenons that, in general, involve many intrinsic binding constants to account for intramolecular and intermolecular binding. However, for our purposes, an equivalent binding constant based on a monomolecular binding scheme should be adequate to provide a first-order estimate of sensor behavior.

We have demonstrated the multivalency effect on $K_{a}^{*}$ in another system-the binding of multivalent antigens to an antibody. $\mathrm{Liu}^{6}$ prepared bovine gamma globulin derivatized with various amounts of dinitrophenol and measured the concentration of dinitrophenol needed to displace the multivalent hapten from a DNP-antibody. The results shown in FIGURE 4 demonstrate that the affinity of the "analog-analyte" (i.e., $K_{a}^{*}$ ) could be changed by several orders of magnitude in this manner.

Another important consideration in the design of biosensors is the response time of the sensor to changes in analyte concentration. One can get an appreciation of the

TABLE 2. Association Constants of Sugars to Con- $A^{a}$

\begin{tabular}{lc}
\multicolumn{1}{c}{ Sugar } & Association Constant \\
\hline Methyl- $\alpha$-D-mannopyranoside & 20,600 \\
Methyl- $\alpha$-D-glucopyranoside & 4,940 \\
Maltose & 2,880 \\
D-fructose & 1,370 \\
D-glucose & 588 \\
Methyl- $\beta$-D-glucopyranoside & 176 \\
\hline
\end{tabular}

${ }^{a}$ Conditions: $\mathrm{pH} 6.2,1 \mathrm{M} \mathrm{NaCl}$, and $2{ }^{\circ} \mathrm{C} .{ }^{10}$ 


\section{Fraction of \\ Free Analyte}

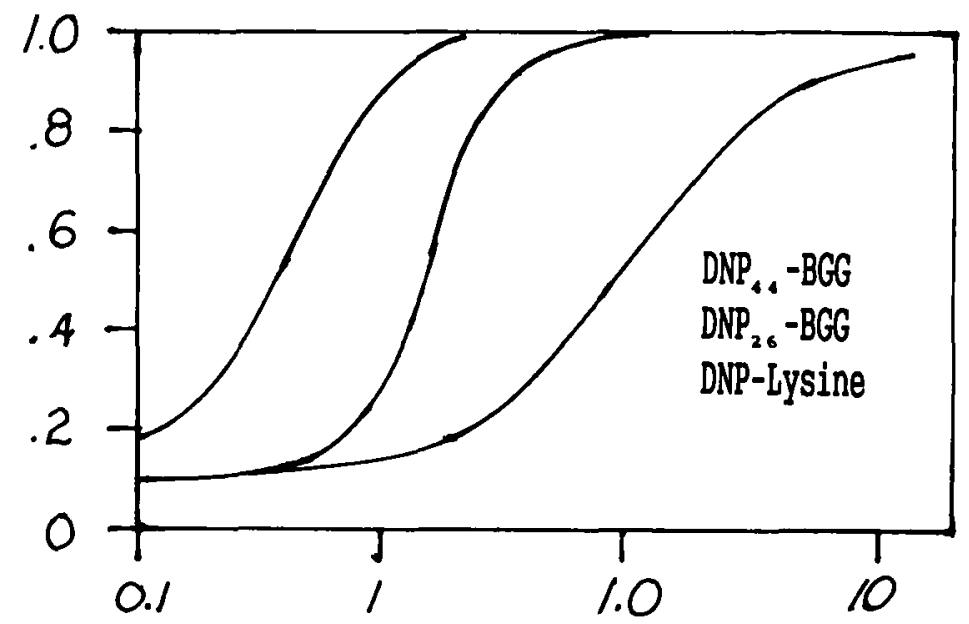

\section{Analyte-Analog Concentration}

FIGURE 4. Effect of multivalency of analog-analyte on the response of a biosensor.

factors involved in the response of systems of this type by individually considering the different physical chemical processes that take place in a sensor of this type. As shown earlier, ${ }^{7}$ both diffusional and chemical processes are occurring. The diffusional response times depend on the dimensions of the device, the membrane structure, and the molecular weight of the species involved. For our glucose sensor (where the reaction between glucose and Con- $A$ is on the order of milliseconds), the diffusional processes dominate and are on the order of five minutes. However, systems utilizing bioreceptors with high binding constants can become rate-limited by the chemical reactions.

Consider the simple case of a monomolecular reaction, that is, the direct method mentioned above:

$$
\mathbf{A}+\mathbf{R}=\mathbf{A}: \mathbf{R}
$$

Then, recalling that in a sensor configuration, the total amount of bioreceptor is conserved, the rate of change in complexed receptor concentration with a sudden change in analyte concentration is given by

$$
d[\mathrm{~A}: \mathrm{R}] / d t-k_{\mathrm{f}}[\mathrm{A}]\left([\mathrm{R}]_{\mathrm{t}}-[\mathrm{A}: \mathrm{R}]\right)-k_{\mathrm{r}}[\mathrm{A}: \mathrm{R}]
$$

In order to obtain an order of magnitude estimate of the response time of the system, we assume the initial state of the system is the condition where the receptor is 
TABLE 3. Association and Dissociation Rate Constants for Enzyme-Ligand Complexes $^{11}$

\begin{tabular}{llccc}
\hline \multicolumn{1}{c}{ Enzyme } & Ligand & $\begin{array}{c}k_{f} \\
\left(\mathrm{M}^{-1} \mathrm{~s}^{-1}\right)\end{array}$ & $\begin{array}{c}k_{r} \\
\left(\mathrm{~s}^{-1}\right)\end{array}$ & $\begin{array}{c}K_{a} \\
\left(\mathrm{M}^{-1}\right)\end{array}$ \\
\hline $\begin{array}{llll}\text { Old yellow enzyme } \\
\text { Malate dehydrogenase }\end{array}$ & FMN & $10^{6}$ & $10^{-4}$ & $10^{10}$ \\
Lactate dehydrogenase & NADH & $10^{8}$ & 10 & $10^{7}$ \\
$\begin{array}{l}\text { Chymotrypsin } \\
\text { Creatine kịnase }\end{array}$ & NADH & $10^{7}$ & 10 & $10^{6}$ \\
$\begin{array}{l}\text { Aspartate } \\
\text { aminotransferase }\end{array}$ & Proflavin & $10^{8}$ & $10^{3}$ & $10^{5}$ \\
\hline
\end{tabular}

one-half saturated, that is,

$$
\mathrm{A}_{0}=K_{\mathrm{a}}^{-1} \text { and }\left[\mathrm{A}: \mathrm{R}_{0}\right]=[\mathrm{R}]_{\mathrm{l}} / 2 \text {. }
$$

Then, for a small increase in analyte concentration, $\Delta \mathbf{A}_{0}$, there will be a first-order response to a new final steady-state reading,

$$
[A: R]_{f}=[R]_{t}(1+\Delta) /(2+\Delta),
$$

with a half-time given by the relationship for small $\Delta$,

$$
t_{1 / 2}=(\ln 2) /\left(2 k_{\mathrm{r}}\right) \text {. }
$$

Therefore, for these assumptions, the kinetic behavior is dominated by the dissociation rate constant for the analyte-bioreceptor complex. Thus, for values of $k_{\mathrm{r}}=10^{4}, 10^{-2}$, 10 , and $10^{2} \mathrm{~s}^{-1}$, the half-times would be on the order of $1 \mathrm{~h}, 0.5 \mathrm{~min}, 0.3 \mathrm{~s}$, and $3 \mathrm{~ms}$, respectively.

The kinetics of biological reactions vary over many orders of magnitude. Some typical values of association and dissociation rate constants for reversible enzymeligand complexes are shown in TABLE 3, along with the association equilibrium constants, which, in reciprocal concentration units, again provide an approximate indication of the range of sensitivity if these reactions were to be used as the basis of a biosensor for the particular ligands indicated in the table.

It is logical to consider what may be the ultimate constraints on the sensitivity and response rate of systems of this sort. Experience with antibodies to haptens has shown that association equilibrium constants from $10^{10}$ to $10^{12}$ can be found, thus indicating that very high levels of sensitivity can be obtained. However, the individual rate constants for association and dissociation must also be considered if a practical sensor with a reasonable response rate is to be fabricated.

In the case of antibodies, it appears that the association rate constant is about the same for all the antibodies and on the order of $10^{-8} \mathrm{M}^{-1} \mathrm{~s}^{-1}$ (see Figure 5). This behavior is consistent with the concept that the association rate in the limit for chemical reactions is diffusion controlled. Then, using the Smoluchowski approximation, the association constant is given by

$$
k_{\mathrm{f}}=4 \pi r\left(D_{\mathrm{A}}+D_{\mathrm{B}}\right) N_{0}
$$


where $r$ is the radius of the molecule, $D_{A}$ and $D_{B}$ are the diffusivities of the two species, and $N_{0}$ is Avogadro's number. For room temperature, this formula gives a value for the association rate constant of about $10^{-9} \mathrm{M}^{-1} \mathrm{~s}^{-1}$. This value is about 10 times faster than the average association rate constant for the DNP antibodies plotted in FIGURE 5. This, though, is expected because the Smoluchowski limit does not account for sterochemical limitations; that is, only a fraction of the binding protein surface is the active site of the bioreceptor.

As an illustration of the limitations of the use of bioreceptors in the equilibriumdisplacement biosensor, the antibodies with the highest sensitivity to DNP in FIGURE 5 have a dissociation constant of about $5 \times 10^{-4} \mathrm{~s}^{-1}$. Using the formula given above, a sensor using these antibodies would have a time constant on the order of 10 minutes, which may be much too slow for "on-line" monitoring.

This apparent limitation, that is, the slow response for systems with high levels of sensitivity, has been the subject of further research. One approach, suggested by Andrade, ${ }^{8}$ is to control the local environment of the bioreceptor so as to change the binding constant and kinetics. This could be achieved by periodically changing the local $\mathrm{pH}$, ionic strength, or hydrophobicity. Andrade proposed to immobilize photosensitive polymers (whose configuration could be changed by illumination) in the vicinity of the bioreceptor. The polymer could be switched from one form to another by light pulses and this could presumably cause rapid dissociation of the ligand-bioreceptor complex. If this approach were successful, the sensor would be periodic rather than continuous in nature; however, this might be satisfactory if the switching rate could be made fast enough.

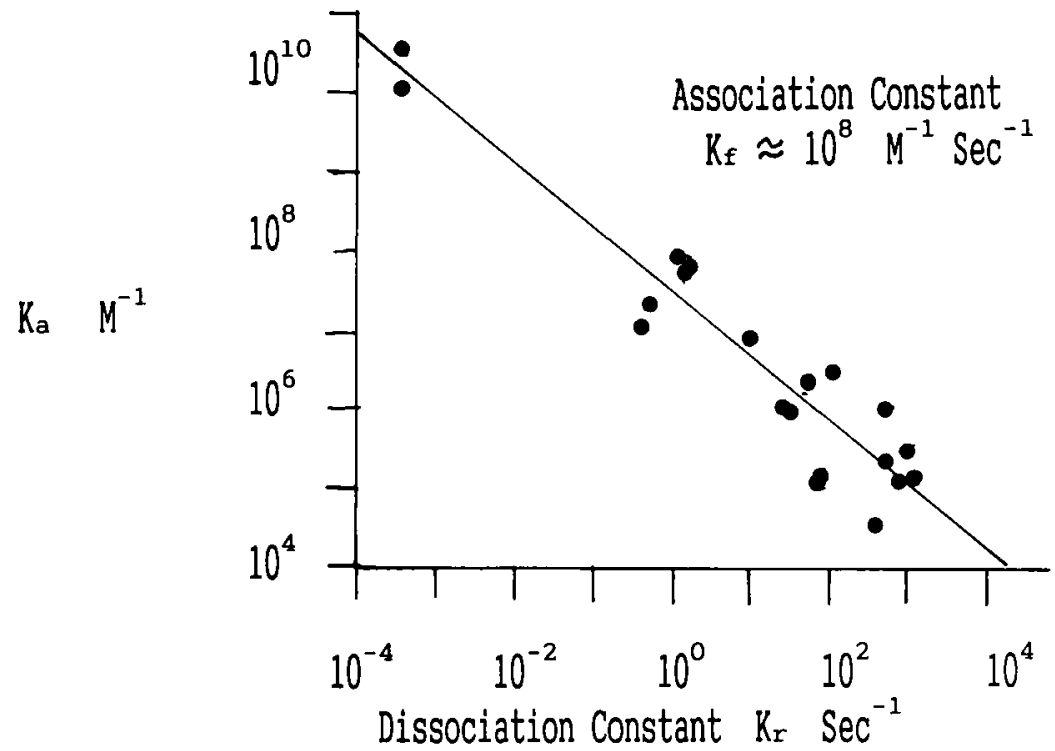

FIGURE 5. Relationship between the association equilibrium constants, $K_{\mathrm{a}}$, and the dissociation rate constants, $k_{r}$, for a series of antibodies to dinitrophenol (DNP). 


\section{SUMMARY}

Bioreceptors can provide the basis for specific and sensitive biosensors. There are many sources of bioreceptors in nature for biochemicals of interest in biotechnology and biomedicine. These bioreceptors (antibodies, enzymes, membrane proteins, binding proteins) can be modified and produced in large quantities using modern biotechnological techniques.

The characteristics of the biosensor can also be fine-tuned by modifying the structure of the analog-analyte, which will also provide several orders of magnitude of range sensitivity with a given bioreceptor.

The ultimate sensitivity of the biosensor may be limited by the dissociation kinetics of the reaction between analyte and bioreceptor because there is a trade-off between sensitivity and sensor response rate to changes in analyte concentration.

\section{REFERENCES}

1. Shaw, E. J., R. A. A. Watson, J. London \& D. S. Smith. 1977. Estimation of serum gentamicin by quenching fluoroimmunassay. J. Clin. Pathol. 30: 562-571.

2. SchultZ, J. S. \& G. SimS. 1979. Affinity sensors for individual metabolites. Biotechnol. Bioeng. Symp. 9: 65-71.

3. MANSOUR1, S. \& J. S. SCHULTZ. 1984. A miniature optical sensor based on affinity binding. Biotechnology 2: 385-390.

4. MansourI, S. 1983. Optical glucose sensor based on affinity binding. Ph.D. thesis. University of Michigan.

5. PERelson, A. S. 1984. Some mathematical models of receptor clustering by multivalent ligands. In Cell Surface Dynamics: Concepts and Models. A.S. Perelson, C. DeLisi \& F.W. Wiegel, Eds.: 223-276. Dekker. New York.

6. LIU, B. \& J. S. SchulTz. 1986. Equilibrium binding in immunosensors. IEEE Trans. Biomed. Eng. 53: 133-138.

7. Schultz, J. S., S. MANSOURI \& I. J. GoldSTEIN. 1982. Affinity sensor: a new technique for developing implantable sensors for glucose and other metabolites. Diabetes Care 5: 245253.

8. Andrade, J., J. N. Lin, J. Herron, M. Reichert \& V. Hlady. 1986. Remote fiber-optic biosensors based on evanescent field fluoroimmunoassay. Paper no. MBTD 10. Amer. Chem. Soc. 192nd Meeting, Anaheim, California (September).

9. Bachas, L. G., C. D. Tsalta \& M. E. MeYerhoff. 1986. Binding proteins as reagents in enzyme-linked competitive binding assays of biological molecules. Biotechniques 4: 4256.

10. So, L. L. \& I. J. GoldSTEIN. 1968. On the number of combining sites on Concanavalin A, the phytohemagglutinin of the jack bean. Biochem. Biophys. Acta 165: 398-404.

11. Adapted from: Hammes, G. G. \& P. R. Schimmel. 1970. In The Enzymes, Vol. 2. P. D. Boyer, Ed.: 67. Academic Press. New York.

12. SchultZ, J. S. 1986. Design of fiber-optic biosensors. In Biosensors, Fundamentals and Applications. A. P. F. Turner, I. Karube \& G. S. Wilson, Eds. Oxford University Press. London/New York.

13. LIU, B. 1986. Characterization of immunosensor binding reactions. Ph.D. thesis. University of Michigan. 